\title{
Shadow Removal using YCBCR and K-Means Clustering
}

\author{
Anuradha Baghel \\ M.Tech Scholar \\ Department of Information Technology \\ S.A.T.I. College, \\ Vidisha (M.P), India
}

\author{
Vismay Jain \\ Assistant Professor \\ Department of Information Technology \\ S.A.T.I College, \\ Vidisha (M.P.),India
}

\begin{abstract}
Shadow physical phenomena observed in natural scenes. Image segmentation, tracking, recognition algorithms to fail or can cause a shadow. In this paper, conducted a research in the field of research on the perception of image complexity and classification of images, remove the shade. The purpose of this paper is two-fold: in the first place, an attempt is to be presented for consideration to overcome some of the fundamental problems of light reflection, color constancy, and at the second place segmentation is applied in place to provide better color information. Experimental results show that the proposed method can detect and eliminate the shadows effectively and maintained the color information properly.
\end{abstract}

\section{Keywords}

Shadow removal, bilateral filter, segmentation, k-means algorithm

\section{INTRODUCTION}

Shadow because of distinctive illuminations regularly causes issues in computer vision and design undertaking. For example, darker regions (brought about by shadows) can present mistaken fragments in segmentation calculations, brilliance changes (created by shadows) diminish the execution of article acknowledgment frameworks, and shape from shading calculations accept that surface brilliance does exclude shadow impacts. In computerized photography, shadows are ordinarily considered as relics in the image that should be uprooted, whether for improving certain parts of the image or just for various reasons. The issue of producing an inborn image that is invariant to illuminations is essential yet difficult, and the late techniques can be isolated into the accompanying three classes.

1) Methods that utilize various images. Weiss [1] determined an inborn reflectance image of a scene given an arrangement of images of the scene under a scope of illumination conditions. Weiss' strategy certainly accepts that the scene is made out of Lambertian surfaces. On the off chance that a surface displays Lambertian reflectance, light falling on it is scattered such that the obvious shine of the surface to an onlooker is the same paying little respect to the eyewitness' point of perspective. Matsushita et al. [2] extended Weiss' technique to handle non-Lambertian scenes as the Lambertian presumption does not hold for true scenes. Matsushita et al. [3] later minimized a regularization capacity that exploits the onesided image subsidiaries to yield reflectance images less affected by shading. These routines produce fruitful results. However, various images obtained under the required conditions are not generally accessible by and by, e.g., for element scenes and moving cameras.
2) Methods that utilize a solitary image however require human connection. Levin and Weiss [4] proposed a client helped system to perform reflection partition and one of its applications is shadow evacuation. Wu et al. [5], [6] defined the shadow development issue as one of vitality minimization guided by client supplied indications as a quad-guide which can be determined effortlessly by the client. Liu and Gleicher [7] developed a shadow-impact free and composition predictable angle field between the shadow and lit region and recuperated the sans shadow image from it by fathoming a Poisson comparison. These routines deliver great results with cautious client help, however are not of completely programmed.

3) Methods that utilization a solitary image without human collaboration. Tappen et al. [8] proposed a learningbased way to deal with discrete reflectance edges and illumination edges in a subordinate image. Despite the fact that this strategy effectively isolates reflectance and shading for a given illumination bearing utilized for preparing, it is not intended for subjective lighting headings. By accepting Lambertian surface, Planckian lighting 1 and narrowband camera sensor, Finlayson and Hordley [9] determined a 1-D illuminant-invariant image. The change of RGB representation to a 1-D grayscale representation diminished the separation between each two surfaces in the image. Finlayson and Drew [10] extended this technique to determine a 2-D inherent image utilizing a fourband camera, and hypothetically demonstrated that $3-\mathrm{D}$ invariant images can be recouped with six-band cameras. The issue with this methodology is that four-band and sixband cameras are seldom utilized. Utilizing edge location to the first and the 1-D illuminant-invariant image.

4) Finlayson and Drew et al. presents a strategy that was conceived for the recuperation of an invariant image from a 3-band color image. The invariant image, initially $1 \mathrm{D}$ greyscale yet here inferred as a $2 \mathrm{D}$ chromaticity, is free of lighting, furthermore has shading evacuated: it shapes a characteristic image that may be utilized as an aide as a part of recuperating color images that are autonomous of illumination conditions. Invariance to illuminant color and power implies that such images are free of shadows, also, to a decent degree. The system formulated finds an inborn reflectivity image in view of suspicions of Lambertian reflectance, around Planckian lighting, and decently narrowband camera sensors. All things considered, the technique functions admirably when these suppositions don't hold. A vital bit of data is the plot for an"invariant bearing" in a log-chromaticity space. 


\section{SHADOW REMOVAL}

Let us recapitulate how this linear behavior with lighting change results from the assumptions of Planckian lighting, Lambertian surfaces, and a narrow band camera. The intensity, then, of each of these three color channels can be described by

$\rho m(x, y)=\delta(x, y) \int E(\lambda, x, y) S(\lambda, x, y) Q m(\lambda) d \lambda$

with the illumination spectral power distribution (SPD) being $\mathrm{E}(\lambda, \mathrm{x}, \mathrm{y})$, the surface spectral reflectance function $\mathrm{S}(\lambda, \mathrm{x}, \mathrm{y})$, camera sensor sensitivity functions $\operatorname{Qm}(\lambda), \mathrm{m} \in r, g, b$, and $\sigma$ Lambertian shading: surface normal dotted into illumination direction.We note that the camera sensor $\operatorname{Qm}(\lambda)$ behaves similar to a Dirac delta function,so we assume that the camera sensitivities are Dirac delta functions:

$\mathrm{Qm}(\lambda)=\mathrm{qm} \delta(\lambda-\lambda m)$

Substituting Eq. (2) into Eq. (1), we obtain

$\rho m(x, y)=\delta(x, y) \int E(\lambda, x, y) S(\lambda, x, y) q m \delta(\lambda-\lambda m) d \lambda$

$\rho m(x, y)=\delta(x, y) E(\lambda, x, y) S(\lambda, x, y) q m$

Then, Eq. (1) becomes simply

$\rho m=\delta E(\lambda m) S(\lambda m) q m$

We use Planck's law to model the illumination spectrum:

$\mathrm{E}(\lambda, \mathrm{T})=\frac{2 \pi h c^{2}}{\lambda m^{5}} \frac{1}{e^{h c / \lambda m K T}-1}$

where $\mathrm{k}=1: 381 \times 10^{-23} \mathrm{~J} / \mathrm{K}$ is Boltzmann's constant,$\lambda$ is the wave- length, $c=3: 0 \times 10^{8} \mathrm{~m} / \mathrm{s}$ is the speed of light, $\mathrm{h}=6: 626$ $\times 10^{-34} \mathrm{JS}$ is Planck's constant, and T is the color temperature. Substituting Eq. (5) into Eq. (4), we obtain

$\rho m=\delta \frac{2 \pi h c^{2}}{\lambda m^{5}} \frac{1}{e^{h c / \lambda m K T}-1} \mathrm{~S}(\lambda \mathrm{m}) \mathrm{qm}$

For the surface reflection model, the surface reflectance is a combination of a diffuse model and a specular model commonly used in computer graphics. There are two kinds of extreme cases: Lambertian reflection and ideal specular reflection. Assume that each image pixel can be treated independently using the method proposed below. $\delta 1$ and $\delta 2$ are respectively factors for Lambertian reflection and specular reflection:

$\rho m=\rho m 1+\rho m 2=(\delta 1+\delta 2 S(\lambda)) \frac{2 \pi h c^{2}}{\lambda m^{5}} \frac{1}{e^{h c / \lambda m K T}-1} \mathrm{qm}$

Taking log of Eq (7) we obtain

$$
\begin{aligned}
& \rho^{\prime} m=\log (\rho m)= \\
& \quad \log \left[\frac{(\delta 1+\delta 2 S(\lambda m) q m)}{\lambda m^{5}}\right]+\log \left(2 \pi h c^{2}\right)-\log \left(e^{h c / \lambda m K T}-1\right)
\end{aligned}
$$

Let $\mathrm{Xm}=\log \left[\delta 1+\delta 2 S(\lambda m) q m / \lambda m^{5}\right], \mathrm{A} 1=\log \left(2 \pi h c^{2}\right)$, $\mathrm{A} 2=h c / k, \mathrm{Ym}=\log \left(e^{A 2 / \lambda m T}-1\right)$ Then, $\mathrm{Eq}(8)$ becomes simply

$\rho^{\prime} m=X m+A 1-\log \left(e^{A 2 / \lambda m T}-1\right)$

where the typical temperature range of most light sources $\mathrm{T} \in[2500,10000]^{0} \mathrm{~K}$.

$\log \left(e^{A 2 / 2 m T}-1\right) \approx \log \left(\left(e^{A 2 / \lambda m T}\right)\right.$
Then Eq.(9) becomes simply

$\rho^{\prime} m=X m+A 1-\frac{A 2}{2 m T}$

The chromaticity of image and the chromaticity of surface component can be defined as below according to the definition of chromaticity:

$\rho^{\prime \prime} m=\frac{\rho^{\prime} m}{\rho^{\prime} r+\rho^{\prime} g+\rho^{\prime} b}$

$X^{\prime} m=\frac{X m}{X r+X g+X b}$

While $\varepsilon \rho^{\prime \prime} m=1, \varepsilon X^{\prime \prime} m=1, m \in r, g, b$

Eqs. (12) and (13) can be reformulated:

$\rho^{\prime} m=\rho^{\prime \prime} m\left(\rho^{\prime} r+\rho^{\prime} g+\rho^{\prime} b\right)$

$\mathrm{Xm}=\mathrm{X}^{\prime} \mathrm{m}(\mathrm{Xr}+\mathrm{Xg}+\mathrm{Xb})$

Substituting Eqs. (14) and (15) into Eq. (11), we obtain

$\rho^{\prime} m=\mathrm{X}^{\prime} \mathrm{m}(\mathrm{Xr}+\mathrm{Xg}+\mathrm{Xb})+\mathrm{A} 1-\frac{A 2}{\lambda m T}$

Let $\mathrm{m}$ be $\mathrm{r}$; $\mathrm{g}$; $\mathrm{b}$ in Eq. (16), and then sum up over three channels, we can obtain

$\rho^{\prime} r+\rho^{\prime} g+\rho^{\prime} b=\mathrm{Xr}+\mathrm{Xg}+\mathrm{Xb}+3 \mathrm{~A} 1-\frac{A 2}{\mathrm{\lambda rT}}-\frac{A 2}{\mathrm{\lambda gT}}-\frac{A 2}{\mathrm{\lambda bT}}$

We can simply express Eq. (17) using $\varepsilon \rho^{\prime}=\lambda^{\prime} r+\lambda^{\prime} g+\lambda^{\prime} b$ and $\varepsilon X m=X r+X g+X b$. Substituting Eq. (15) into Eq. (18), we can obtain

$\mathrm{T}=\frac{\left[X^{\prime} m\left(\frac{1}{\lambda r}+\frac{1}{\lambda g}+\frac{1}{\lambda b}\right)-\frac{1}{\lambda m}\right] A 2}{\rho^{\prime} m-X^{\prime} m \varepsilon \rho^{\prime}+\left(3 X^{\prime} m-1\right) A 1}$

At last, substituting Eq. (19) into Eq. (11), then, we obtain

$\rho^{\prime} m=X m+A 1-\frac{\rho^{\prime} m-X^{\prime} m \varepsilon \rho^{\prime}+\left(3 X^{\prime} m-1\right) A 1}{\left[\frac{X^{\prime} m}{\lambda m}\left(\frac{1}{\lambda r}+\frac{1}{\lambda g}+\frac{1}{\lambda b}\right)-1\right]}$

Firstly, let $\lambda$ 'm be a constant and we can get an intrinsic image through computing from Eq. (20). However, the chromaticity is different from pixel to pixel. Based on this observation, we modify the improved bilateral filter to compute the edge. Bilateral filtering is a non-linear filtering technique introduced by Tomasi et al. [16]. It extends the concept of Gaussian smoothing by weighting the filter coefficients with their corresponding relative pixel intensities.

\section{PROPOSED WORK}

Another methodology for image segmentation by applying kmeans calculation is utilized. In image segmentation, clustering calculations are exceptionally well known as they are instinctive and are additionally simple to actualize. The Kmeans clustering calculation is a standout amongst the most generally utilized calculation. Here we utilize color-based segmentation strategy that uses K-means clustering procedure.The $\mathrm{k}$-means calculation is an iterative system used to segment an image into $\mathrm{k}$ regions. The standard K-Means calculation produces precise segmentation comes about just when connected to images characterized by homogenous areas regarding texture and color following no nearby requirements are connected to force spatial coherence. At to 
begin with, the pixels are bunched in view of their color and spatial components, where the clustering procedure is refined. At that point the bunched blocks are converged to a particular number of locales. This methodology subsequently gives a feasible new solution for image segmentation which may be useful in image recovery.

\subsection{Flow Chart for Proposed Work}

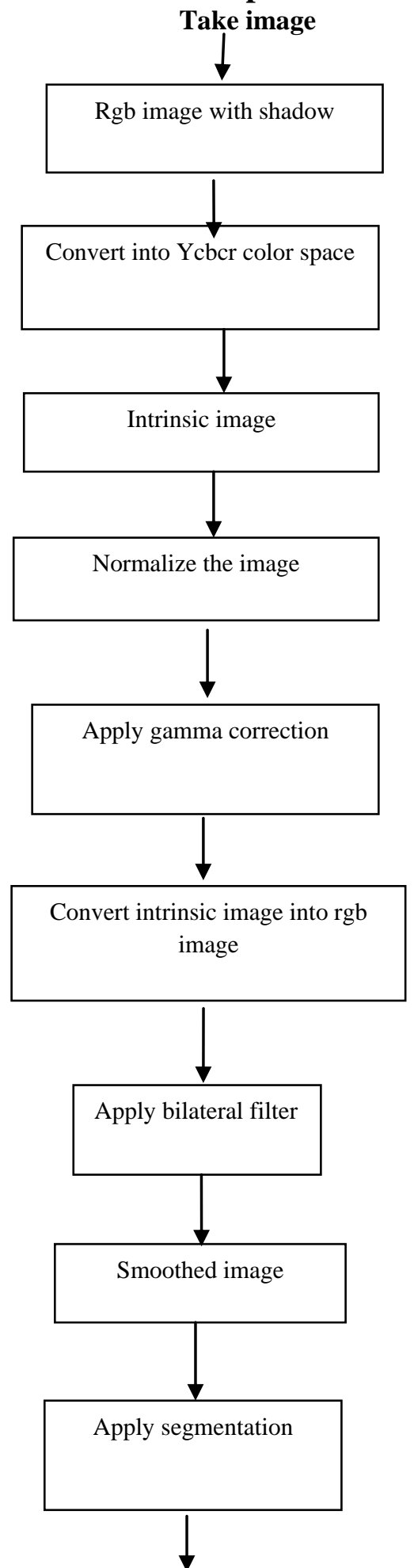

\section{Output Image}

\subsection{K-Means Clustering Algorithm}

Let $X=\{x 1, x 2, x 3, \ldots \ldots \ldots, x n\}$ be the set of data points and $\mathrm{V}=\{\mathrm{v} 1, \mathrm{v} 2, \ldots \ldots, \mathrm{vc}\}$ be the set of centers.

1) Choose 'c' cluster centers randomly.

2) Calculate the distance between each data point and cluster centers.

3) Allocate the data point to the cluster center whose distance from the cluster center is minimum of all the cluster centers.

4) Recalculate the new center of cluster using the equation:

$$
v_{i}=(1 / c i) \sum_{j=1}^{c i} x i
$$

Where, 'ci' shows the number of data points in $i^{\text {th }}$ cluster.

5) Recalculate the distance between each data points and new obtained cluster centers.

6) If no data point was reassigned then stop, else repeat from step 3 .

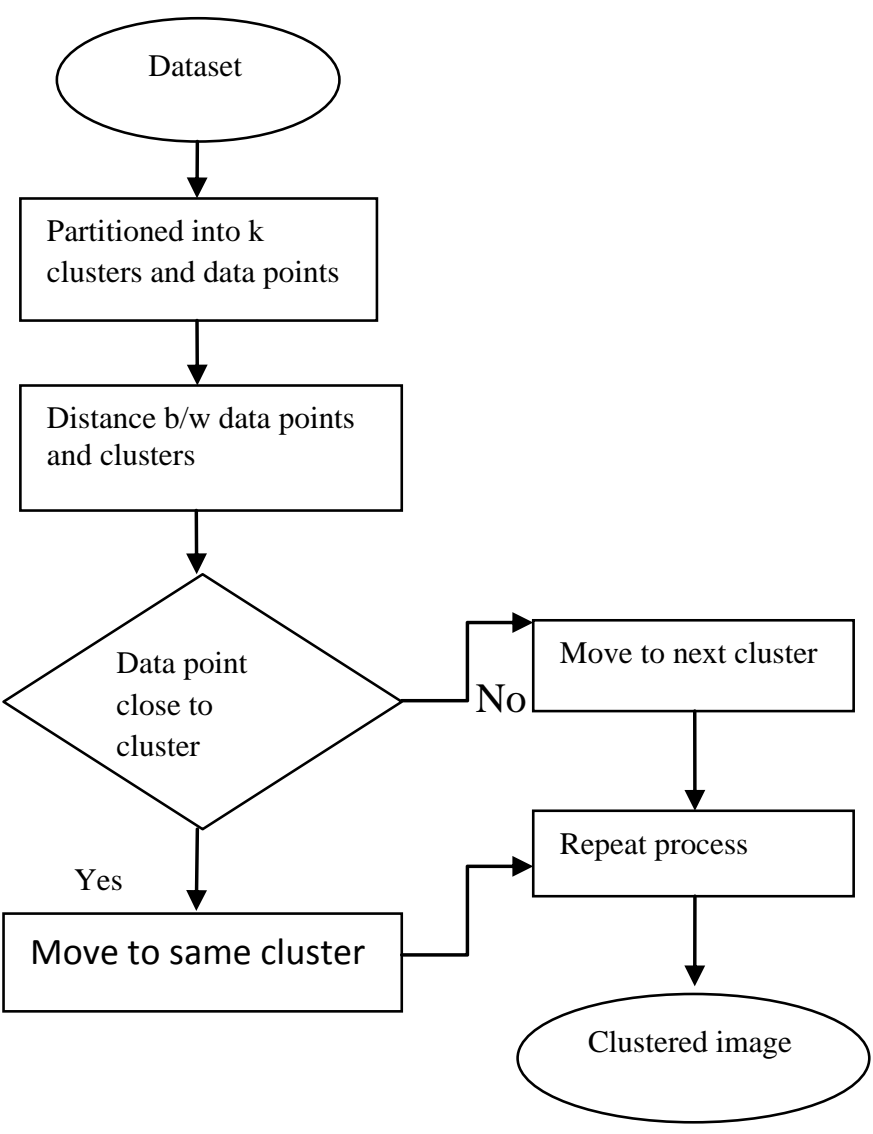

Flow Chart For K-Means Clustering

\section{EXPERIMENTAL RESULTS}

In this section, some experiments were performed on real images 


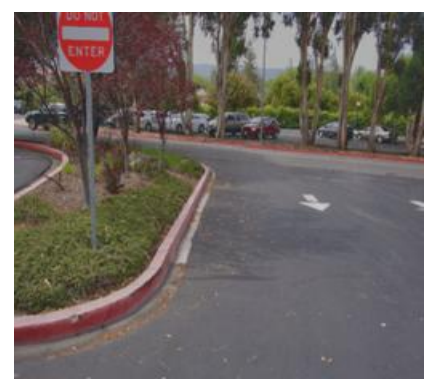

Figure 1

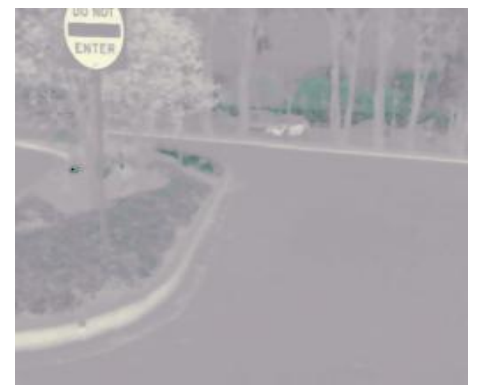

Figure 2

Figures shows the Application in real image processing. Figure 1 shows the original image (original image is loaded from http://www.cs.cityu.edu.hk/_qiyang/), figure 2 shows

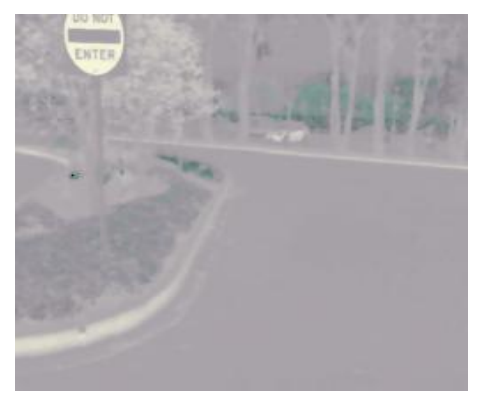

Figure 3

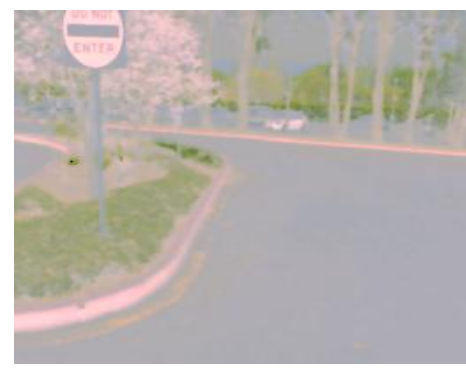

Figure 4

intrinsic image with a constant, figure 3 shows image after applying bilateral filter and figure 4 shows the final our shadow-free image.

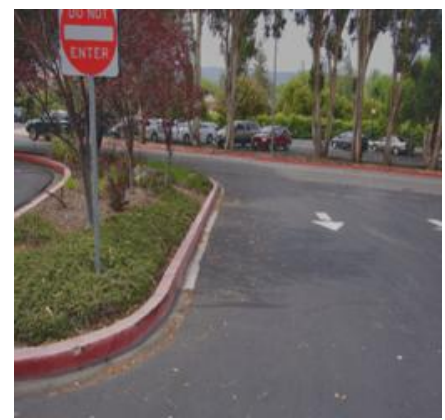

Figure 1

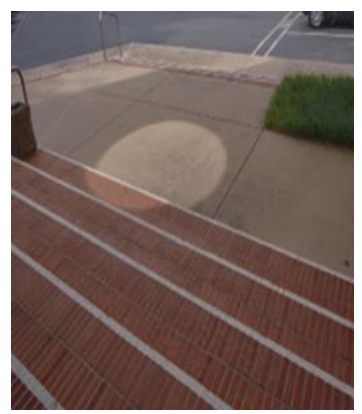

Figure 1

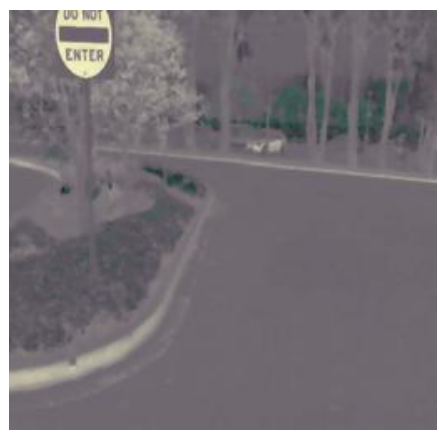

Figure 2

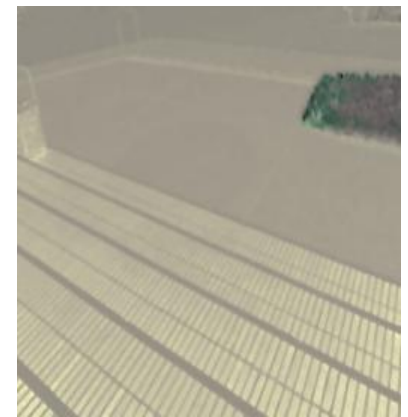

Figure 2

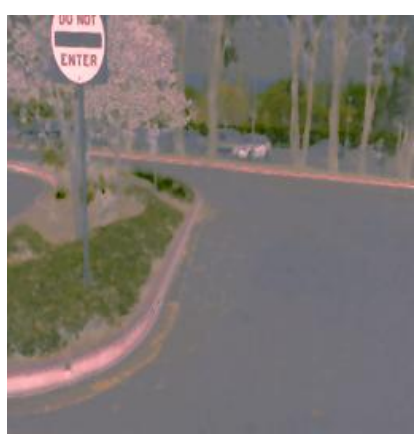

Figure 3

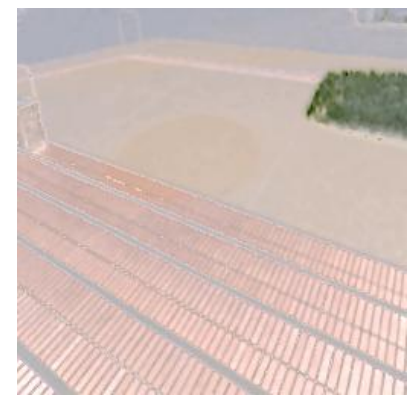

Figure 3 


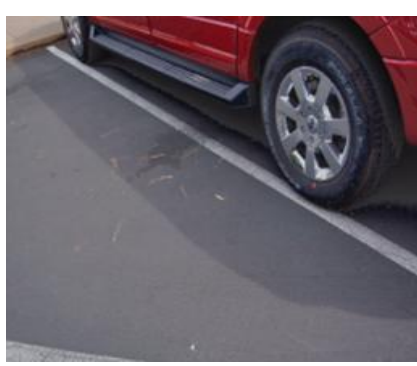

Figure 1

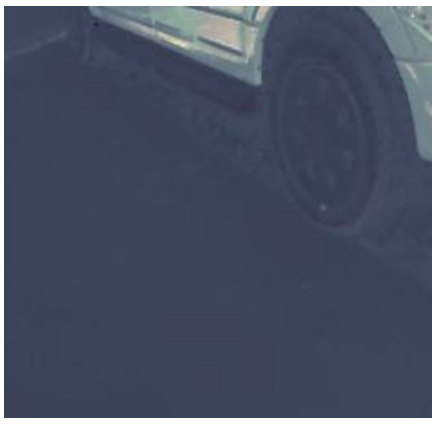

Figure 2

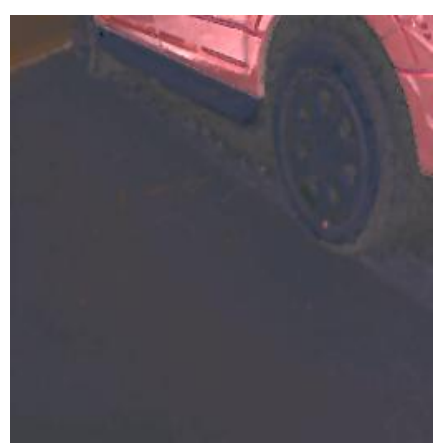

Figure 3
Some example images. Each row shows results for a different image. The first column shows the original image with shadow. The second column is the shadow-free image
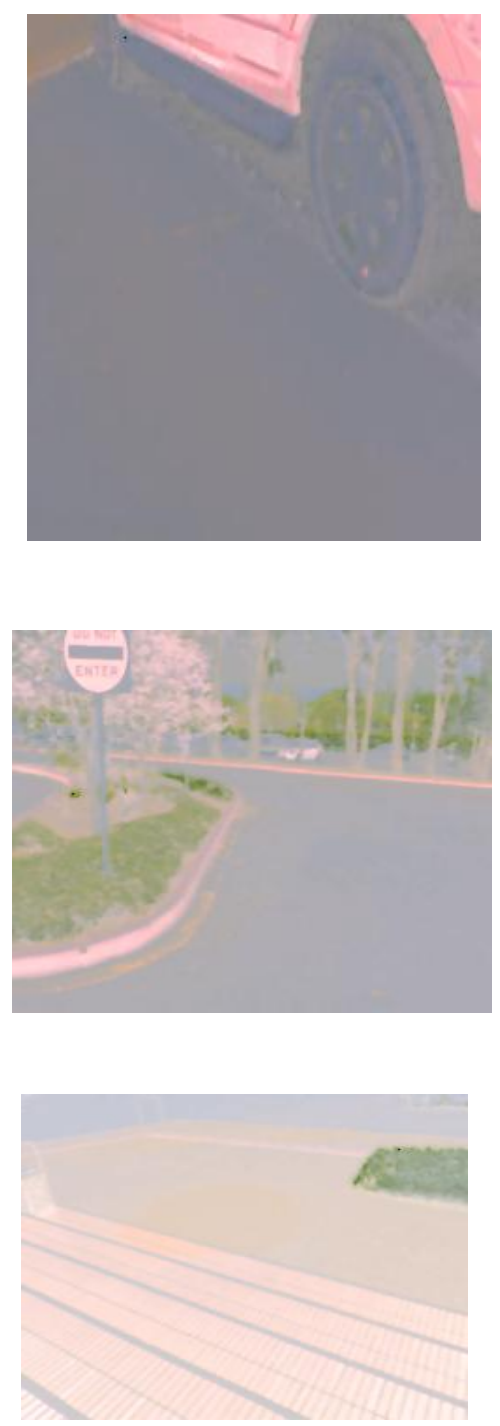

Above images shows the comparison between the previous and our proposed work the first column shows the shadow free image from X. Zhu [15], the second column shows the (intrinsic image). The final Column shows the shadow free image of proposed model.
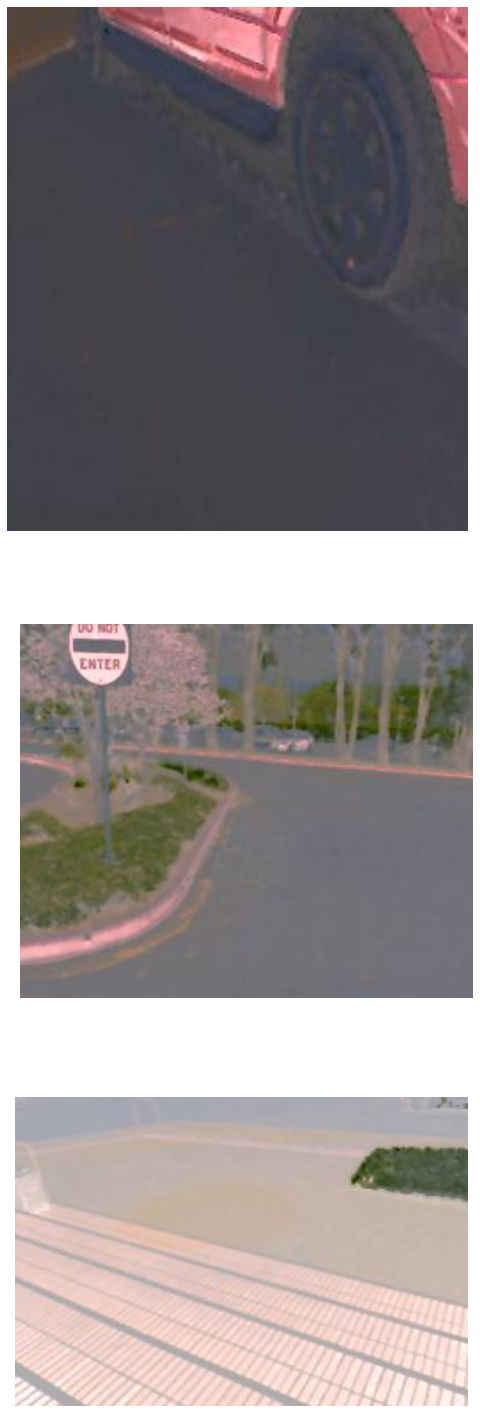

images of our proposed method. The right images are more clear than left side images. 


\section{CONCLUSION}

In this paper, the system to locate an intrinsic reflectivity image was exhibited, which depends on suppositions of Lambertian reflectance, approximately Planckian lighting, narrow band camera sensors and the idea of bilateral filtering for edge-saving smoothing. In light of these issues, a shadow removal algorithm was presented in this paper. A few cases working on genuine images are incorporated into this paper, in which shadows are all evacuated. Division by utilizing kmeans clustering gives the image which is more clear than the past technique. The outcome goes for adding to an exact and more dependable image which can be utilized as a part of finding tumors, measure tissue volume, face recognition, unique mark recognition and in finding an article plainly from a satellite image. The benefit of K-Means calculation is basic and entirely effective. It functions admirably when clusters are not all around isolated from one another. This could be happen in web images. We proposed a structure of unsupervised clustering of images taking into account the color feature of the image.

\section{REFERENCES}

[1] Y.Weiss, "Deriving intrinsic images from image sequences", in: Proceedings of International Conference on ComputerVision,2001, pp.68-75.

[2] Q. Yang, K.-H. Tan, " Shadow removal using bilateral filtering”, IEEE Trans. Image Process. 21(October(10)) (2012) 4361-4368

[3] Y.Matsushita, S. Lin, etal. ,"Estimating intrinsic images from image sequences with biased illumination", in: Proceedings of European Conference on Computer Vision, 2004, pp.274-286.

[4] A. Levin and Y. Weiss, "User assisted separation of reflections from a single image using a sparsity prior," IEEE Trans. Pattern Anal. Mach. Intell., vol. 29, no. 9, Sep. 2007, pp. 1647-1654.

[5] T.-P. Wu and C.-K. Tang, "A Bayesian approach for shadow extraction from a single image," in Proc. Int. Conf. Comput. Vis., 2005, pp. 480-487

[6] T.-P. Wu, C.-K. Tang, M. S. Brown, and H.-Y. Shum, "Natural shadow matting," ACM Trans. Graph., vol. 26, no. 2, pp. 357-366, 2007.
[7] F. Liu and M. Gleicher, "Texture-consistent shadow removal," in Proc. Eur. Conf. Comput. Vis., 2008, pp. 437-450.

[8] M. F. Tappen, W. T. Freeman, and H. Adelson, "Recovering intrinsic images from a single image," IEEE Trans. Pattern Anal. Mach. Intell., vol. 27, no. 9, Sep. 2005, pp. 1459-1472.

[9] G.D. Finlayson, S.D. Hordley, C.Lu,M.Drew, On the removal of shadows from images, IEEE Trans. Pattern Anal.Mach.Intell.28(January(1))(2006),pp.59-68.

[10] G. D. Finlayson and M. S. Drew, "4-sensor camera calibration for image representation invariant to shading, shadows, lighting, and specularities," in Proc. Int. Conf. Comput. Vis., 2001, pp. 473-480.

[11] G.D.Finlayson ,S.D .Hordley, M.S.Drew , "Removing shadows from images", in: Proceedings of European Conference on Computer Vision,vol.IV,2002, pp. 823836.

[12] G.D.Finlayson,M.Drew, C.Lu, "Intrinsic images by entropy minimization",in: Proceedings of the Eighth European Conference on Computer Vision, May 2004,pp.582-595.

[13] Y. Matsushita, S. Lin, S. B. Kang, and H.-Y. Shum, "Estimating intrinsic images from image sequences with biased illumination," in Proc. Eur. Conf. Comput. Vis., 2004, pp. 274-286.

[14] C. Lu, M.S. Drew, and G.D. Finlayson, "On the removal of shadows from images," IEEE Tran. on Pattern Analysis and Machine Intelligence (PAMI), 2006, pp. 134-142.

[15] Xia Zhu n, Renwen Chen, Huakang Xia and Piaoyan Zhang, "Shadow removal based on YCbCr color space," in proc. Journal of Neurocomputing, vol. 151,2014,pp. 252-258.

[16] C. Tomasi, R. Manduchi, "Bilateral filtering for gray and color images, in: Proce. Int. Conf. Comput. Vis., 1998, pp. 839-846. 